\title{
Characterization of Burkholderia pseudomallei protein BPSL1375 validates the Putative hemolytic activity of the COG3176 N-Acyltransferase family
}

\author{
Laziana Ahmad ${ }^{1}$, Teng Loong Hung ${ }^{1}$, Nor Azurah Mat Akhir ${ }^{1,2}$, Rahmah Mohamed ${ }^{1,3}$, Sheila Nathan ${ }^{1}$
} and Mohd Firdaus-Raih ${ }^{1,4^{*}}$ (D)

\begin{abstract}
Background: There are still numerous protein subfamilies within families and superfamilies that do not yet have conclusive empirical experimental evidence providing a specific function. These proteins persist in databases with the annotation of a specific 'putative' function made by association with discernible features in the protein sequence.

Results: Here, we report the characterization of one such protein produced by the pathogenic soil bacterium Burkholderia pseudomallei, BPSL1375, which provided evidence for putative hemolysins in the COG3176 family to have experimentally validated hemolytic activity. BPSL1375 can be classified into the N-acyltransferase superfamily, specifically to members of the COG3176 family. Sequence alignments identified seven highly conserved residues (Arg54, Phe58, Asp75, Asp78, Arg99, Glu132 and Arg135), of which several have been implicated with Nacyltransferase activity in previously characterized examples. Using the 3D model of an $\mathrm{N}$-acyltransferase example as a reference, an acyl homoserine lactone synthase, we generated 3D structure models for mutants of six of the seven N-acyltransferase conserved residues (R54, D75, D78, R99, E132 and R135). Both the R99 and R135 mutants resulted in a loss of hemolytic activity while mutations at the other five positions resulted in either reduction or increment in hemolytic activity.
\end{abstract}

Conclusions: The implication of residues previously characterized to be important for $\mathrm{N}$-acyltransferase activity to hemolytic activity for the COG3176 family members of the N-acyltransferase provides validation of the correct placement of the hemolytic capability annotation within the N-acyltransferase superfamily.

Keywords: Burkholderia pseudomallei, Hemolysin, BPSL1375, N-acyltransferase

\section{Background}

Burkholderia pseudomallei is a soil bacterium that causes melioidosis, a deadly disease endemic to South East Asia and Northern Australia [1]. This bacterium is recognized as a potential category B biothreat agent due to its aerosol infectivity and severe course of infection

\footnotetext{
* Correspondence: firdaus@mfrlab.org

'School of Biosciences and Biotechnology, Faculty of Science and Technology, Universiti Kebangsaan Malaysia, 43600 UKM, Bangi, Selangor, Malaysia

${ }^{4}$ Institute of Systems Biology, Universiti Kebangsaan Malaysia, 43600 UKM, Bangi, Selangor, Malaysia

Full list of author information is available at the end of the article
}

[2]. Pathogenesis of B. pseudomallei remains poorly understood despite a number of previous studies having described the expression of several virulence factors, particularly extracellular products with proteolytic, lipolytic and hemolytic activity [3, 4], the Type VI secretion system [5] and the capsule [6]. A toxin that deamidates and subsequently inactivates helicase activity of the translation initiation factor (eIF4a) has been reported [7]. Other aspects of this bacterium's genome has also been explored including the existence of small RNAs [8] and methylation of genomic DNA by restriction modification systems [9]. Nevertheless, much of the genome 
remains annotated as genes encoding hypothetical proteins.

Most bacterial pathogens have the capacity to acquire nutrients and minerals from the infected hosts [10]. Free iron may generate damaging free radicals and therefore is typically maintained at low concentrations within the host. Bacterial pathogens are known to secrete extracellular proteins that lyse erythrocytes in order to acquire the free iron released from heme [11]. Hemolysins, one such class of secreted proteins, are exotoxins that have lytic activity against erythrocytes and are often implicated as virulence factors. These proteins also allow bacteria to evade the immune system by escaping from phagosomes [12]. Many hemolysins act by forming pores on the target cell's membrane while others act through surfactant-like or enzymatic mechanisms [13].

Hemolysins have been implicated in a variety of complications arising from bacterial infections, ranging from intestinal tract disease to septicemia. Hemolysins produced by Escherichia coli have been associated with enterotoxicity and septicemia [14], whilst those secreted by Vibrio vulnificus [15], Aeromonas hydrophila [16] and Yersinia ruckeri [17] have been detected in high concentrations during fatal infections. Radke et al. [18] reported that Listeria monocytogenes listeriolysin disrupts lysosomal and phagosomal membranes to allow for bacterial escape into the host cytoplasm to initiate bacterial replication. Hemolysins have also been previously reported for members of the B. cepacia complex $[19,20]$ and it is known that different strains produced different hemolysins with differing functions. The Japanese clinical strain had been reported to produce a hemolysin called cepalysin that is capable of forming pores up to $30 \mathrm{~nm}$ in diameter [21]. Two separate hemolysins have been described for U.S clinical isolates with a size of $72 \mathrm{kDa}$ and $22 \mathrm{kDa}$ [22]. The hemolysins of British isolates are capable of inducing apoptosis and degranulation of mammalian phagocytes [19] and thus quite distinct from both the Japanese and the U.S strains due to its lack of pore forming capacity.

A number of studies have reported the expression of several B. pseudomallei heat-stable and heat-labile toxins that exhibited cytolytic and hemolytic activity [23-25]. Elevated levels of either cytotoxicity or hemolytic activity have also been detected in supernatant extracts of stationary growth phase cultures [24]. However, it is still unclear if this hemolytic phenotype is the result of a single enzyme or a result of the combined outcomes from the reactions of different proteins.

Open reading frames (ORFs) annotated as hypothetical proteins make up almost $25 \%$ of the B. pseudomallei genome [26]. Cruz-Migoni et al. [7] reported a genomewide project to characterize $B$. pseudomallei hypothetical proteins in an attempt to discover proteins with novel functions, which may include putative virulence factors. In this paper, we report the characterization of the protein encoded by the coding region bpsl1375 and provide definitive evidence of its ability to lyse erythrocytes. In silico analysis revealed sequence level similarities to members of the $\mathrm{N}$-acyltransferase superfamily (Conserved Domains Database ID: cl00357) from the COG3176 group of the Clusters of Orthologous Groups (COGs) database. Members of COG3176 have previously been annotated as putative hemolysins.

\section{Results}

\section{BPSL1375 is a member of the $\mathrm{N}$-acyltransferase} superfamily

A systematic approach to characterize the functions of coding regions annotated as hypothetical proteins by Holden et al. [27] resulted in the extraction of all such sequences followed by resubmission for sequence alignment based analysis. This included targeted searches for previously reported virulence and pathogenicity factors such as various hemolysins from other Gram negative bacteria. One of these searches identified motifs previously observed in the hemolysin of Ralstonia eutropha within the coding region of an ORF originally annotated as encoding the hypothetical protein BPSL1375 by Holden et al. [27]. A database search of the BPSL1375 sequence in GenBank revealed that the sequence contained domains that could be remotely associated with the N-acyltransferase (NAT) superfamily and shared sequence similarity to other proteins that were also annotated as hypothetical proteins. The NAT superfamily (Conserved Domains Database ID: cl00357) consists of various enzymes that characteristically catalyze the transfer of an acyl group onto its substrate. Among the known members of this superfamily are proteins that have been annotated as putative hemolysins (COG3176). The PATRIC database's [28] entry for BPSL1375 has been updated to take into account this known homology to hemolysin and thus carries a revised annotation for BPSL1375 as 'putative hemolysin'.

This homology to the NAT superfamily enabled us to refine our BLASTP database searches using the higher quality curated datasets in SWISSPROT/UniProt [29] and the Protein Data Bank (PDB) [30]. The UniProt search yielded 13 hits, all with scores lower than 40, E values of 0.07 or higher and sequence identities of $25 \%$ and lower. Two of the hits were for acyl-homoserine-lactone (AHL) synthase, a well characterized member of the NAT superfamily. The BLASTP search against the PDB also yielded hits with scores lower than 30 , E values of 1.7 or higher and sequence identities of $37 \%$ or lower. Again, we were able to observe a hit for an acyl-homoserine-lactone synthase, specifically, the sequence for the crystal structure of Pseudomonas aeruginosa LasI [31] with the PDB code 1RO5. 
To identify highly conserved regions such as residues that may be potential active sites or binding motifs, we carried out multiple sequence alignment of the BPSL1375 sequence against 26 other sequences from the COG3176 family and representatives from the lysophospholipid acyltransferase (LPLAT) family to which AHL synthase belongs. From the alignments, we identified 7 residue positions - Arginine 54 (R54), Phenylalanine 58 (F58), Aspartic Acid 75 (D75), Aspartic Acid 78 (D78), Arginine 99 (R99), Glutamic Acid 132 (E132) and Arginine 135 (R135) that were conserved in all 27 sequences (Fig. 1a). The equivalent residue numbers for the LasI sequence are provided in Fig. 1b. Six of these residues (R54, D75, D78, R99, E132 and R135) carried charged side chains while F58 is a hydrophobic amino acid.

\section{BPSL1375 and its orthologs are conserved in Burkholderia}

\section{spp.}

We then investigated whether BPSL1375 is common to all Burkholderia spp. and twelve orthologs were discovered in other Burkholderia species with available genome sequences. These Burkholderia spp. orthologs were mainly annotated as either hemolysin-like proteins or ornithineacyl $\mathrm{N}$-acyltransferases. We further explored this conservation by analyzing the presence and arrangement of the bpsl1375 orthologs and the flanking coding regions in the other Burkholderia species. We were able to match twelve orthologous sequence regions within the Burkholderia genus although seven of these appear to have possibly undergone various levels of rearrangement (Fig. 2).

\section{Scanning electron microscopy reveals evidence of hemolysis by BPSL1375}

The potential for BPSL1375 to have hemolytic activity as suggested by the sequence alignment was investigated by incubating purified recombinant BPSL1375 (Additional file 1) with sheep erythrocytes. The expression and purification of recombinant BPSL1375 is discussed in more detail below. Scanning electron microscopy (Fig. 3) revealed that sheep erythrocytes treated with the recombinant BPSL1375 displayed crenated shapes with cytoplasmic blebbing on the membrane (Fig. 3b) compared

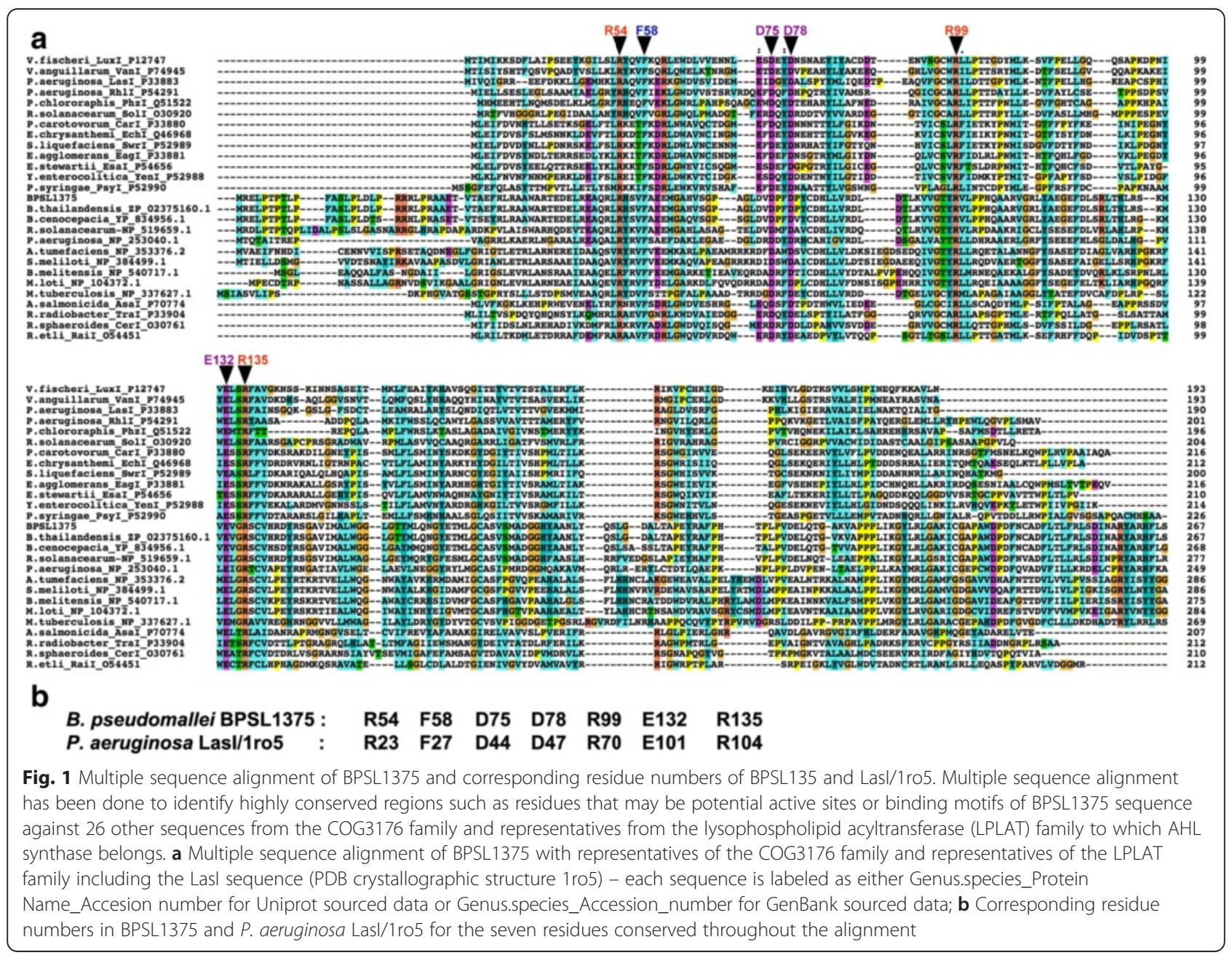




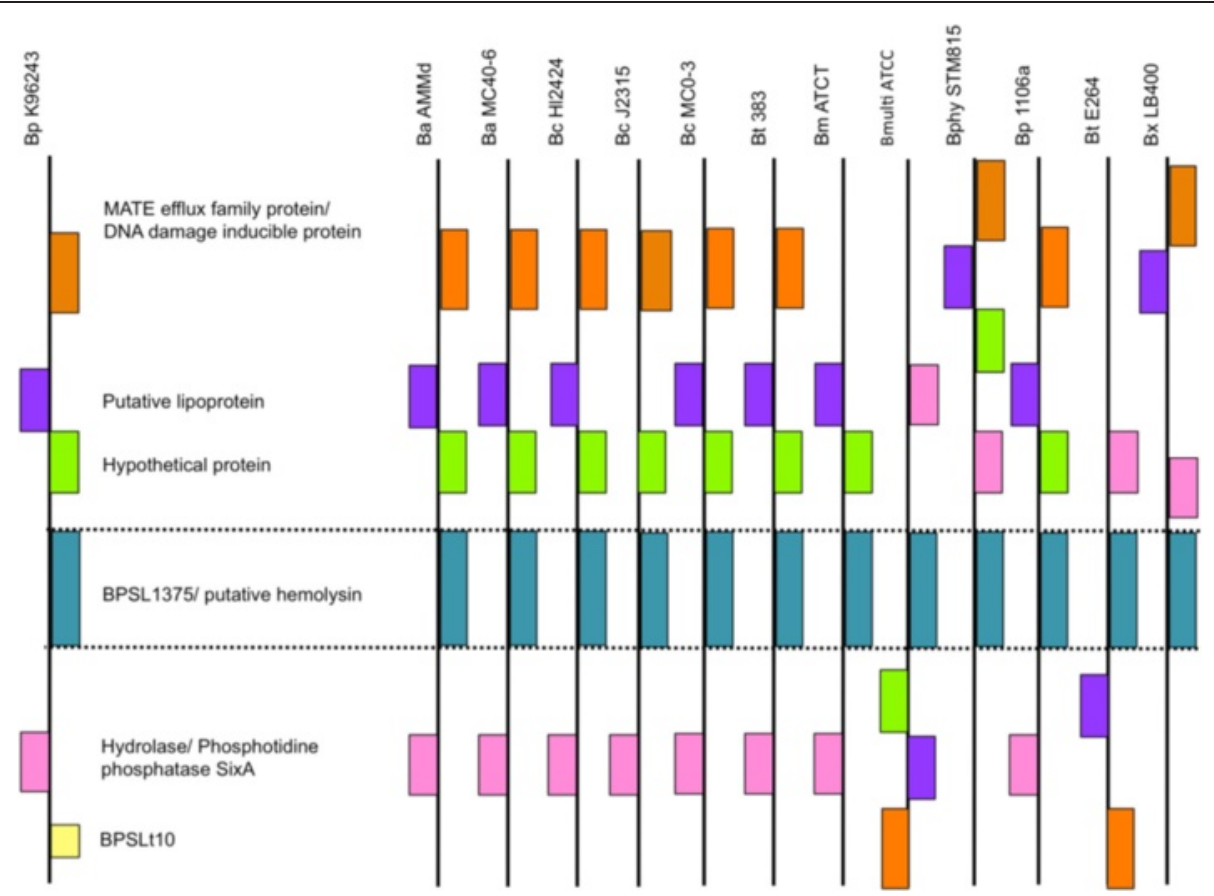

Fig. 2 Conservation of bps/1375 and neighboring genes in other members of the Burkholderia genus. The conservation of bpsl1375 matched twelve orthologous sequence regions within the Burkholderia genus although seven of these appear to have possibly undergone various levels of rearrangement. Bp: B. pseudomallei; Ba: B. ambifaria; BC: B. cenopacia; Bt: B. thailandensis; Bm: B. mallei; B. multi ATCC: B. multivorans ATCC17616; Bphy: B. phymatum; Bx: B. xenovorans

to untreated erythrocytes that presented the usual biconcave discs appearance (Fig. 3a), nevertheless, blebbing was not as notable as the positive control for lysis (Fig. 3c). Recombinant E. coli carrying the bpsl1375 gene (see below) that were grown on blood agar plates also showed clear hemolytic zones (Fig. 3d). Confirmation of BPSL1375 hemolytic activity then led us to perform modeling and generation of recombinant mutants to enable the determination of key residue(s) required for hemolytic activity.

\section{Modeling and structural analysis}

The importance of the seven conserved residues identified by multiple sequence alignment of BPSL1375 with other available AHL synthases was initially investigated using the crystal structure of $P$. aeruginosa LasI (PDB: 1RO5) as reference. The corresponding conserved residues of BPSL1375 in LasI are R54/R23, F58/F27, D75/ D44, D78/D47, R99/R70, E132/E101 and R135/R104 (Fig. 1b). The structural study of 1RO5 indicated that these residues were involved in the formation of the substrate binding pocket in an electrostatic cluster of the molecule [31], thus suggesting that these residues play key roles in supporting the proper structure and formation of the binding site.

Each of the seven conserved residues was substituted in silico with residues from the same group of amino acids such as $\mathrm{R}$ with $\mathrm{K}$ and $\mathrm{D}$ with $\mathrm{E}$ in order to maintain the protein's general fold as well as the local conformations at the substitution sites [32]. Visual analysis using Chimera molecular graphics [33] revealed the consistency of the local conformation for the substituted residues compared to the original residues indicating the suitability of the substitution in conserving the position and orientation of the original residue in 3D space (Fig. 4). We further investigated changes in the number of hydrogen bonds with the neighboring residues that resulted from the substitution. The substitution of R23K reduced the number of hydrogen bonds formed from 5 to 4 (Fig. 4a), D44E from 4 bonds to 3 (Fig. 4b), D47E from 4 bonds to 2 bonds (Fig. 4c), R70K from 5 bonds to 3 (Fig. 4d), E101D from 6 bonds to 5 bonds (Fig. 4e) and R104K with 5 bonds reduced to 3 bonds (Fig. 4f). With these mutant models as a guide, we expect the changes in the number of hydrogen bonds to change the interactions between the residues involved, thus, possibly affecting the overall catalytic properties of the molecule. In the next section, we demonstrate the effect of each substitution on the hemolytic activity of BPSL1375.

\section{Characterization of BPSL1375 and site directed mutants} In order to characterize the BPSL1375 protein, the bpsl1375 gene was cloned from a clinical isolate (B. pseudomallei D286) available in our laboratory. 


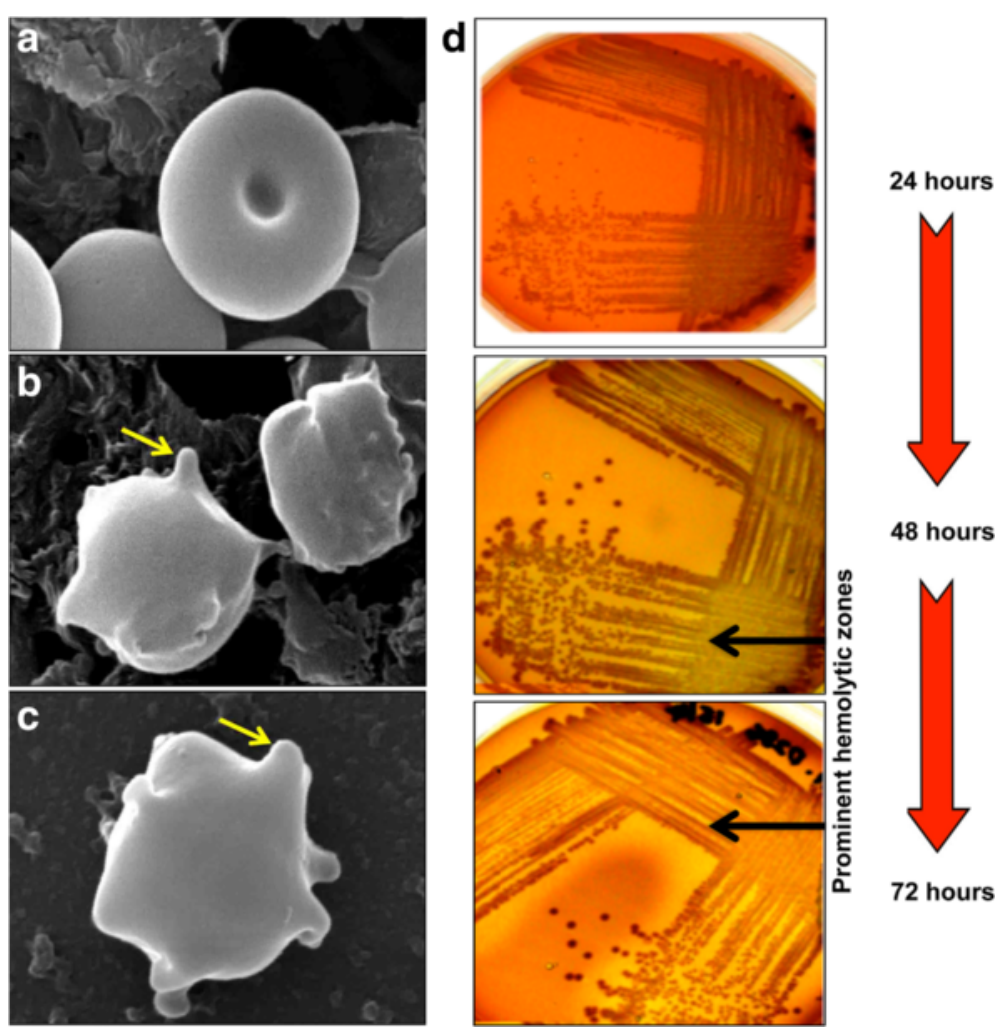

Fig. 3 A comparison between the electron micrographs of cytoplasmic blebbing on the membrane and recombinant $E$. coli carrying the bpsl1375 gene grown on blood agar plates. Scanning electron microscopy revealed that sheep erythrocytes treated with the recombinant BPSL1375 displayed crenated shapes with cytoplasmic blebbing on the membrane (a) compared to untreated erythrocytes that presented the usual biconcave discs appearance (b), nevertheless, blebbing was not as notable as the positive control for lysis (c). Recombinant $E$. coli carrying the bps/1375 gene (see below) that were grown on blood agar plates also showed clear hemolytic zones (d). a Normal biconcave disc shape of untreated erythrocytes in PBS; b BPSL1375 treated erythrocytes showing the presence of discrete blebs (yellow arrow) on the erythrocyte; and $\mathbf{c}$ erythrocytes in sterile distilled water that also show the blebs (yellow arrow) as a positive control for lysis. $\mathbf{d}$ Recombinant E. coli bps/1375colonies grown on trypticase soy agar with $5 \%$ sheep blood after 24,48 and $72 \mathrm{~h}$ of incubation at $37^{\circ} \mathrm{C}$ showing clear hemolytic zones

The primers used for the work reported here (Table 1) were designed using the available B. pseudomallei K96243 genome sequence. The amplified 819 bp ORF of bpsl1375, encoding 272 amino acids, was successfully ligated into the expression vector pET200/D-TOPO. The $37 \mathrm{kDa}$ BPSL1375 protein was expressed by E. coli BL21 (DE3) and purified by affinity chromatography.

The E. coli clones expressing recombinant BPSL1375 produced clear hemolytic zones around the colonies on trypticase soy agar containing $5 \%$ sheep's blood (Fig. 3d). Quantification of BPSL1375 hemolytic activity on $3 \%$ sheep blood suspension for $1 \mathrm{~h}$ at $37{ }^{\circ} \mathrm{C}$ revealed up to $26.9 \%$ hemolysis. To investigate the contribution of the conserved amino acids towards the observed BPSL1375 hemolytic activity, site directed mutagenesis was performed on R54, F58, D75, D78, R99, E132 and R135. Of the seven conserved positions, six residues were successfully substituted: R54K, D75E, D78E, R99K, E132D and R135K. All six successful site directed substitutions were then confirmed by Sanger sequencing.
The R135K and R99K substitutions resulted in a complete loss of hemolytic activity indicating that these residues may be critical for the hemolytic activity observed for BPSL1375 (Table 2). The other three substitutions of E132D, D75E and R54K resulted in a non-significant decrease of hemolytic activity. The hemolysis activity of E132D was recorded at $22 \%$ whereas D75E and R54K hemolysis were at $18 \%$ and $14 \%$ respectively. This implied that although these residues are conserved within the COG3176 family members and most likely important for BPSL1375 hemolytic activity, they may not be directly involved in the catalytic reaction or interactions associated with hemolysis and perhaps, have substrate binding roles associated to substrate specificity determination. We also noted that the hemolytic activity of the D78E mutant increased up to 2.3 fold higher than the wild-type (Table 2). For the work reported here, a single sampling to quantify hemolysis by BPSL1375 was conducted at $1 \mathrm{~h}$. Future studies should investigate the hemolytic activity of BPSL1375 and the site directed mutants over time. 


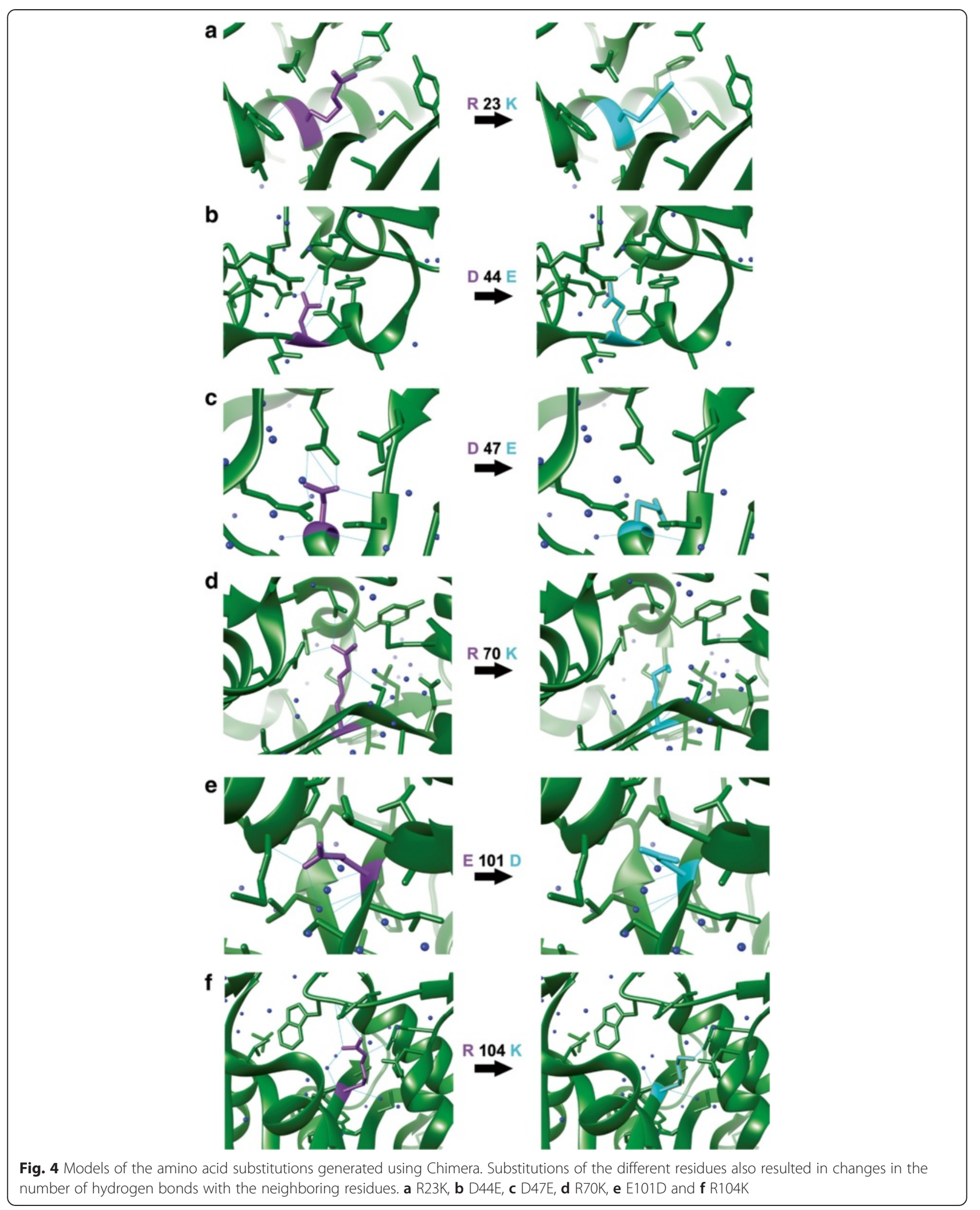


Table 1 Primers used for PCR amplification of bps/1375 and site directed mutagenesis of bps/1375

\begin{tabular}{ll}
\hline Primer name & Primer Sequence (5'-3') \\
\hline BPSL1375_F & CACCATGCGAGAACTGCCGA \\
BPSL1375_R & GCGCGGCAGCGGATCGCTCA \\
BPSL1375_R54K_F & GAAGCGCAGCGGCTGAAGCACAGTGTGTTCGCC \\
BPSL1375_R54K_R & GGCGAACACACTGTGCTTCAGCCGCTGCGCTTC \\
BPSL1375_D75E_F & GGCCTCGACGTCGAGCCGTTCGACC \\
BPSL1375_D75E_R & GGTCGAACGGCTCGACGTCGAGGCC \\
BPSL1375_D78E_F & GACGTCGATCCGTTCGAGCCGTACTGC \\
BPSL1375_D78E_R & GCAGTACGGCTCGAACGGATCGACGTC \\
BPSL1375_R99K_F & TGAAGGTCGTCGGCACCTATAAGGTGCTGCCGCC \\
BPSL1375_R99K_R & GGCGGCAGCACCTTATAGGTGCCGACGACCTTCA \\
BPSL1375_E132D_F & CGAAGATGGTCGATGTCGGCCGCTCGT \\
BPSL1375_E132D_R & ACGAGCGGCCGACATCGACCATCTTCG \\
BPSL1375_R135K_F & GATGGTCGAAGTCGGCAAGTCGTGCGTGCATCGCG \\
BPSL1375_R135K_R & CGCGATGCACGCACGACTTGCCGACTTCGACCATC \\
\hline
\end{tabular}

\section{Hemolysis induced by BPSL1375 wild type and mutant proteins are temperature dependent}

The BPSL1375 recombinant wild type and mutant proteins were incubated individually in erythrocyte suspensions to observe hemolysis at four different temperatures; $4{ }^{\circ} \mathrm{C}, 20^{\circ}, 37^{\circ} \mathrm{C}$ and $60{ }^{\circ} \mathrm{C}$ (Fig. 5a). Incubation was fixed at $60 \mathrm{~min}$ for all reactions except for the $60{ }^{\circ} \mathrm{C}$ sample which was limited to $15 \mathrm{~min}$. The mixtures were centrifuged and the level of hemoglobin released was measured at $545 \mathrm{~nm}$. Poor hemolysis was observed for all samples at $4{ }^{\circ} \mathrm{C}$, including the positive control B. pseudomallei lysate. However, hemolysis at $20{ }^{\circ} \mathrm{C}$ was the highest for B. pseudomallei lysate, BPSL1375, BPSL1375/D78E, BPSL1375/ R99K and BPSL1375/R135K (Fig. 5a) whilst the highest hemolysis at $37{ }^{\circ} \mathrm{C}$ was noted for BPSL1375/E132D. Incubation at $60{ }^{\circ} \mathrm{C}$ abolished the activity of all samples except

Table 2 Quantitation of hemolytic activity of the recombinant wild type and mutant BPSL1375 proteins

\begin{tabular}{ll}
\hline Protein / Control & $\begin{array}{l}\text { Hemolysis } \\
\text { Activity (\%) }^{\text {a }}\end{array}$ \\
\hline Distilled water & 100 \\
B. pseudomallei D286 lysate & 80 \\
Wild type recombinant BPSL1375 & 27 \\
Recombinant R54K & 14 \\
Recombinant D75E & 18 \\
Recombinant D78E & 61 \\
Recombinant R99K & 7 \\
Recombinant E132D & 22 \\
Recombinant R135K & 5 \\
PBS & 9 \\
\hline
\end{tabular}

${ }^{\mathrm{a}}$ Incubation with erythrocytes for $60 \mathrm{~min}$ at $37^{\circ} \mathrm{C}$ for $B$. pseudomallei lysate. The presence of a heat labile hemolysin has been reported for $B$. pseudomallei although it has not been specifically identified [23]. Our results suggest that BPSL1375 as wellas the mutants are heatlabile, and BPSL1375 may therefore be the previously uncharacterized heat labile hemolysin of B. pseudomallei first reported by Ashdown and Koehler [23].

\section{BPSL1375 is inhibited by high erythrocyte concentrations}

The amount of hemolysin needed to lyse a single erythrocyte molecule was determined by incubating a fixed quantity of B. pseudomallei lysate, BPSL1375 and the mutant proteins with different concentrations (0.2 to $2.5 \%)$ of erythrocytes. According to Oberley et al. [34], one molecule of hemolytic protein is sufficient for lysis and therefore the amount of hemoglobin released by a constant amount of protein is proportional to the erythrocyte concentration until a plateau is reached. Therefore, if more than one molecule of protein is required for lysis, the amount of hemoglobin released should increase to a peak and then decrease. After $1 \mathrm{~h}$ at $37^{\circ} \mathrm{C}$, the mixtures were centrifuged and the amount of hemoglobin released into the supernatant was read at $545 \mathrm{~nm}$. The B. pseudomallei lysate lysed erythrocytes at all concentrations of sheep blood while for BPSL1375 and its mutants, the percentage of hemolysis decreased as erythrocyte concentrations increased (Fig. 5b).

These results suggest that BPSL1375 employs a multihit hemolytic mechanism that is similar to the hemolysins of other pathogenic bacteria such as B. cereus [35], Gardenella vaginalis [36], Streptococcus suis and Synechoctus sp. [37]. This mechanism is explained by the ability of a single toxin to bind only to a limited quantity of cells to induce hemolysis and is inhibited in the presence of a high ratio of erythrocytes to toxin molecule [38]. Therefore, keeping the hemolysin concentration constant and increasing the number of erythrocytes suggests that the optimal ratio is not achieved due to the presence of excess erythrocytes. For instance, Inoue et al. [38] described that two to five molecules of streptolysin $\mathrm{O}$ and Clostridium perfringens delta toxin are required per erythrocyte to induce hemolysis. This characteristic was maintained in BPSL1375 and all of the mutants where similar reduction in hemolytic activity was observed at higher erythrocyte concentrations.

\section{Discussion}

Our characterization of the B. pseudomallei protein BPSL1375 that was originally annotated as a hypothetical protein by Holden et al. [27] but subsequently revised to 'putative hemolysin' in the PATRIC database, has enabled this protein to be classified as a member of the N-acyltransferase (NAT) superfamily that includes several putative hemolysins, other hypothetical proteins 

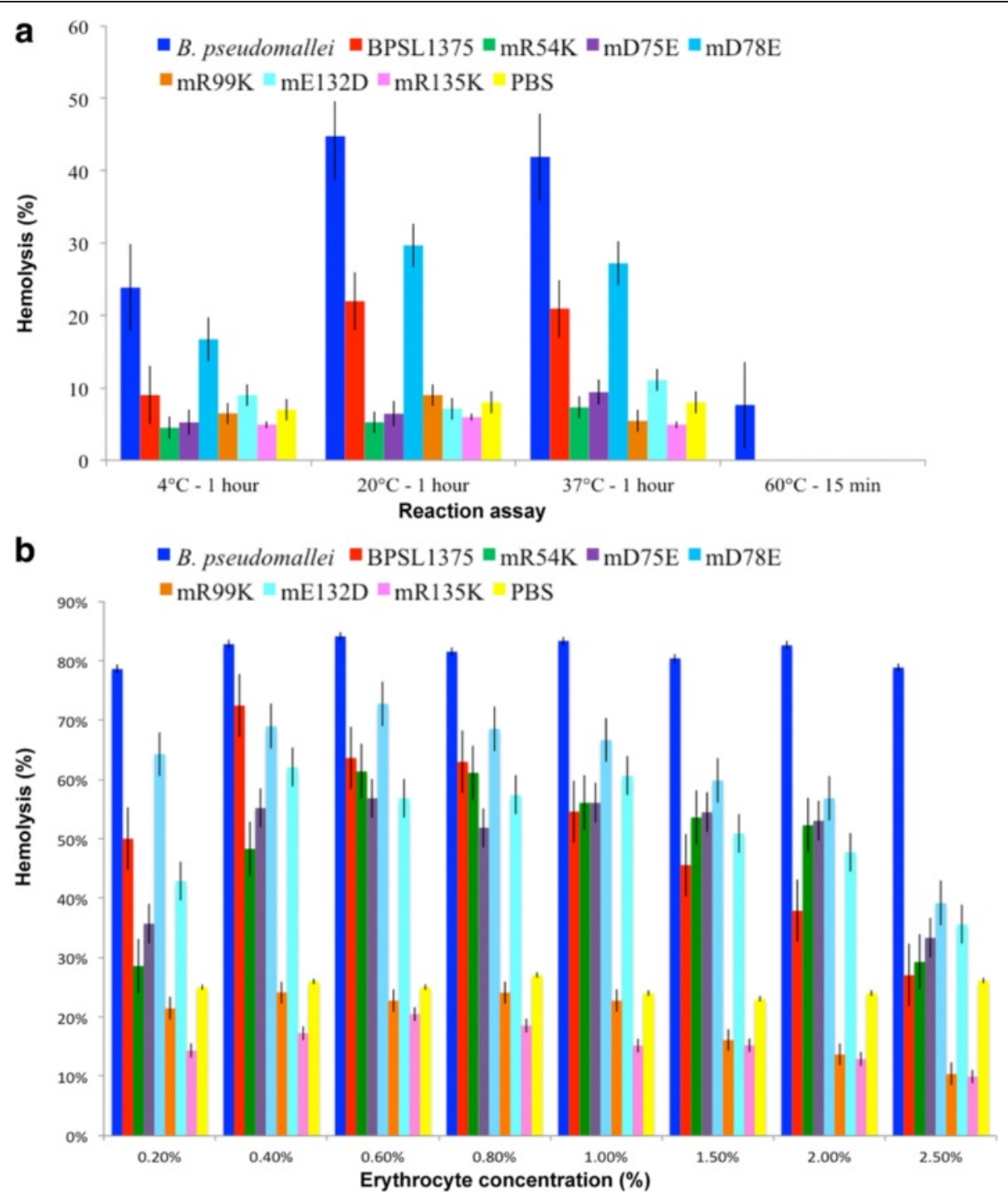

Fig. 5 Hemolytic activity of the wild type and mutant proteins. The hemolytic activity was carried out on $1 \%$ rabbit erythrocyte solution at different temperatures $\mathbf{a}$ and at different erythrocyte concentrations ranging from 0.2 to $2.5 \% \mathbf{b}$ after 60 min incubation at $37^{\circ} \mathrm{C}$. 'B. pseudomallei' refers to B. pseudomallei lysate;'BPSL175' refers to the recombinant protein; mutants are denoted by the scheme "m_Original residue_Residue number_Substituted residue"; PBS refers to phosphate buffered saline

and AHL synthases - namely LasI of Pseudomonas aeruginosa. The BPSL1375 sequence can be further grouped into the COG3176 cluster with members that contain hemolysin-like domains. This hemolytic potential was validated by hemolysis assays and site directed mutants. The hemolytic activity demonstrated by the expression of BPSL1375 (Fig. 3d) is evidence that the protein is the actual structural hemolysin and that BPSL1375 is not merely a regulator of hemolytic activity. To our knowledge, this is the first member of the NAT superfamily with experimental evidence of hemolytic activity.

Multiple sequence analysis to discover BPSL1375 amino acids that were shared with its homologs revealed seven conserved residues (R54, F58, D75, D78, R99, E132 and R135) that are likely important in the protein's function. Cross-referencing of these residues with the observations reported by Gould et al. [31] for the LasI crystal structure revealed that these residues are known to be crucial for the functionality of LasI. These equivalent residues in LasI were deemed to be essential for AHL synthesis and contribute towards the stability of intra-structural interactions with possible catalytic functions. Additionally, the equivalent residue for E101 (corresponding to E132 in BPSL1375) is bound to two commonly conserved water molecules in AHL synthase that is known to partake in the enzyme's catalytic mechanism [31]. 
Based on our modeling of the LasI structure (PDB ID: 1RO5), the most suitable amino acid substitutions that preserved the function of the conserved residues were R54K, F58W, D75E, D78E, R99K, E132D and R135K. These substitute amino acids were selected because they can maintain a similar 3D space and amino acid sequence characteristic to that of the wild type protein. The substitution of $\mathrm{R}$ with $\mathrm{K}$ and $\mathrm{D}$ with $\mathrm{E}$ were carried out to minimize the disturbance of protein fold and structural characteristics, either locally or globally. In this study, the substituted residues were visually scrutinized using Chimera to show the reduction and addition of hydrogen bonds that resulted from the changes, suggesting that the resulting mutants of these residues may show different levels of hemolytic activity.

Consequently, site directed mutagenesis of these seven conserved residues resulted in six successful substitutions and each mutant displayed different degrees of hemolytic capacity. The mutants R99K and R135K showed a complete loss of hemolytic activity and interestingly the D78E mutant showed a significant 2.3 fold increase in hemolytic activity. Although the exact molecular mechanisms for the hemolysis we observed is not yet known, the differences between the degree of hemolysis for each mutant can provide important clues with regards to the contribution of these residues in molecular interactions that result in hemolytic activity. The complete loss of hemolytic activity for mutants of R99 and R135 highlighted that these residues must play a crucial role in the activity of BPSL1375.

Our investigations into the hemolytic activity of BPSL1375 and the six mutants on different erythrocyte concentrations revealed that hemolytic activity decreased with an increase in the ratio between the protein molecule and erythrocyte thus implying that one erythrocyte required a certain number of 'hits' by the protein to induce lysis, a mechanism known as 'multi-hits'. This mechanism has also been observed in Synechocystic sp. hemolysin, a cyanobacterial toxin [37].

\section{Conclusions}

In this study, a B. pseudomallei open reading frame identified using the BpK96243 genome sequence [27] that was initially annotated to encode a hypothetical protein, was characterized as an $\mathrm{N}$-acyltransferase that exhibits hemolytic activity. Genetic manipulation of the residues associated with $\mathrm{N}$-acyltransferase activity resulted in marked detectable variations of hemolytic activity. Although the exact mechanism that resulted in the differences in the exhibited hemolytic activity could not be pinpointed, this study demonstrated the highly useful utility that modeling of amino acid substitutions of available well characterized high resolution structures in the PDB can be reliably transferred to a homolog with very low sequence similarity and thus enabling functional characterization of the remote homolog. Based on our results, R99 and R135 are most likely crucial for function due to the complete loss of activity resulting from their substitution and therefore likely to be among the most highly conserved residues for this family of hemolytic N-acyltansferase (COG3176 family). Our work also demonstrated that BPSL1375 and the mutants possess a multi-hit hemolytic activity towards erythrocytes and that this $\mathrm{N}$-acyltransferase enzyme is not heat stable.

\section{Methods}

\section{Sequence analysis}

Sequence homologs of BPSL1375 were retrieved using BLASTP searches (http://blast.ncbi.nlm.nih.gov) against the following databases: GenBank refseq, SWISSPROT/ UniProt [29], Protein Data Bank (PDB) [30] and the Burkholderia Genome Database [39]. Multiple sequence alignments were performed with ClustalW [40].

\section{Protein structure modeling and analysis}

Models were visualized and analyzed using Chimera [33]. Modeling of mutations, structure comparisons and model refinements were also carried out using Chimera

\section{Strains, vectors, enzymes and chemicals}

B. pseudomallei strain D286 was previously isolated from a fatal melioidosis case at Kuala Lumpur Hospital [41]. A stock culture of this strain was obtained from the Pathogen Laboratory, Faculty of Science and Technology, Universiti Kebangsaan Malaysia. E. coli BL21(DE3) and pET200/D-TOPO were obtained from Invitrogen, USA and other kits as well as reagents were obtained from standard commercial resources.

\section{Primer design}

The ORFs for B. pseudomallei K96243 were generated using ARTEMIS 13.0. Corresponding primers were then designed for the ORF identified as bpsl1375 (Table 1). An additional CACC sequence (5'-CACCATGCGAGAACTGCCGA-3') was added to the 5'- end to allow direct cloning into the expression vector. The primers (Table 1) for Quik-Change site directed mutagenesis were designed using a program provided by Stratagene.

\section{Genomic DNA preparation}

B. pseudomallei D286 was plated on Ashdown selective agar plates. A single colony was inoculated into Brain Heart Infusion broth (Pronadisa, Spain) and grown overnight at $37{ }^{\circ} \mathrm{C}$; the bacterial cells were harvested and the cell pellet was dissolved in TE buffer $(10 \mathrm{mM}$ Tris- $\mathrm{HCl}$, 1 mM EDTA, pH 7.5). The pellet was treated with Proteinase $\mathrm{K}(20 \mathrm{mg} / \mathrm{ml})$ and incubated at $37{ }^{\circ} \mathrm{C}$ for $1 \mathrm{~h}$. 
CTAB-NaCl (5 M NaCl) (Sigma, USA) was added and the incubation was continued at $65{ }^{\circ} \mathrm{C}$ for $20 \mathrm{~min}$. $B$. pseudomallei genomic DNA extraction was carried out according to standard protocols as described by Richards et al. [42]. Purification of genomic DNA was carried out according to the standard phenol chloroform method and precipitated using 0.6 volume of isopropanol.

\section{Amplification and cloning of bps/1375}

DNA amplification by polymerase chain reaction (PCR) was carried out in a final volume of $50 \mu$ containing 10 $\mathrm{X}$ Expand High Fidelity buffer with $15 \mathrm{mM} \mathrm{MgCl}_{2}$ (Sigma Chemical, USA), $10 \mathrm{mM}$ dNTP (Sigma Chemical, USA), $200 \mathrm{ng} / \mu \mathrm{l} \mathrm{B}$. pseudomallei genome, $25 \mu \mathrm{M}$ forward and reverse primers, $3.5 \mathrm{U} / \mu \mathrm{l}$ High Fidelity Taq polymerase (Roche, German) and $\mathrm{dd}_{2} \mathrm{O}$. The amplification was performed over 30 cycles of denaturation at $94{ }^{\circ} \mathrm{C}$ for $1 \mathrm{~min}$, annealing at $67{ }^{\circ} \mathrm{C}$ for $1 \mathrm{~min}$ and elongation at $72{ }^{\circ} \mathrm{C}$ for $1 \mathrm{~min}$. A final extension step was carried out for $10 \mathrm{~min}$ at $72{ }^{\circ} \mathrm{C}$. The amplified products were cloned into pET200/D-TOPO according to the manufacturer's recommendation (Invitrogen, USA). The gene orientation and sequence fidelity were verified by Sanger DNA sequencing.

\section{Construction of mutants}

Single residue mutations were produced using the Stratagene QuikChange site-directed mutagenesis kit. The wild type pET200-bpsl1375 construct was used as a DNA template. The mutations were confirmed by automated DNA sequencing. Six mutants were produced: R54K, D75E, D78E, R99K, E132D and R135K.

\section{Expression and purification of BPSL1375 and its related mutants}

Recombinant bpsl1375 and the corresponding mutants were transformed into E. coli BL21 Star (DE3) according to the kit manufacturer's recommendations (Invitrogen, USA). The bacterial cells were grown on LB (Pronadisa, Spain)-Kanamycin $(30 \mu \mathrm{g} / \mathrm{ml})$ agar plates for $16 \mathrm{~h}$ at $37^{\circ}$ C. A single colony was inoculated into $5 \mathrm{ml} \mathrm{LB-}$ Kanamycin $(30 \mu \mathrm{g} / \mathrm{ml})$ and incubated at $30{ }^{\circ} \mathrm{C}$ overnight with shaking at $250 \mathrm{rpm}$. The overnight culture was subcultured into $50 \mathrm{ml}$ of LB-Kanamycin $(30 \mu \mathrm{g} / \mathrm{ml})$ and grown at $30{ }^{\circ} \mathrm{C}$ with shaking at $250 \mathrm{rpm}$. At $\mathrm{OD}_{600}=0.5$, expression was induced with $1 \mathrm{mM}$ IPTG (Promega, USA) for $6 \mathrm{~h}$ at $30^{\circ} \mathrm{C}$. Cells were harvested and protein extraction procedures were performed at $4{ }^{\circ} \mathrm{C}$. The cell pellet was resuspended in $1 \mathrm{~mL}$ PBS buffer, sonicated for $10 \mathrm{~s}$ at $50 \mathrm{~Hz}$ and then centrifuged at $3200 \mathrm{~g}$ for $30 \mathrm{~min}$. The supernatant containing soluble protein was collected and stored at $-20{ }^{\circ} \mathrm{C}$. His-tagged protein purification was performed using a HisTrap ${ }^{\mathrm{TM}}$ HP column (GE Healthcare). The column was washed with wash buffer
(50 mM NaH $\mathrm{PO}_{4}, 300 \mathrm{mM} \mathrm{NaCl}, 50 \mathrm{mM}$ imidazole, $\mathrm{pH}$ 7.4) and eluted with elution buffer $(50 \mathrm{mM}$ $\mathrm{NaH}_{2} \mathrm{PO}_{4}, 300 \mathrm{mM} \mathrm{NaCl}, 300 \mathrm{mM}$ imidazole, $\mathrm{pH}$ 7.4). The eluted protein fractions were concentrated using Vivaspin 6 (MW $10 \mathrm{kDa}$ ) (Vivascience, Germany). Protein concentration was determined using the bicinchoninic acid method (Sigma Chemical, USA) and analyzed by $12 \%$ SDS-PAGE electrophoresis followed by Western immunoblotting with anti-mouse IgG conjugated with HRP (Invitrogen, USA). C

\section{Ethics statement and consent}

Blood samples from rabbit and sheep were generously provided by the Animal House Facility, Universiti Kebangsaan Malaysia (UKM). Blood was drawn in accordance with the UKM animal ethics guideline formulated based on the guidelines of the National Health and Medical Research Council of Australia. The experiments were approved by the UKM Animal Ethics Committee (UKMAEC) under approval number FST/SBB/2010/ SHEILA/24-AUGUST/320. All animals were maintained in a positive pressure environment at $20-25{ }^{\circ} \mathrm{C}$, subjected to a $12 \mathrm{~h} \mathrm{light/dark} \mathrm{cycle} \mathrm{and} \mathrm{fed} \mathrm{with} \mathrm{a} \mathrm{protein-}$ enriched diet and water ad libitum. No human subjects were used in the experiments reported in this paper.

\section{Preparation of rabbit erythrocyte suspensions}

Rabbit whole blood in anticoagulant citrate dextrose was centrifuged at $750 \mathrm{~g}$ for $10 \mathrm{~min}$. Plasma and white blood cells were discarded by removing the supernatant. Erythrocytes were washed with PBS three times following centrifugation at $750 \mathrm{~g}$ for $10 \mathrm{~min}$.

\section{Measurement of hemolytic activity}

Rabbit erythrocyte suspensions were adjusted to $4 \%$ with PBS. The purified proteins and B. pseudomallei lysates were adjusted to $10 \mu \mathrm{g} / \mathrm{ml}$. Then, $0.1 \mathrm{ml}$ of this solution was incubated with $0.1 \mathrm{ml}$ of $4 \%$ erythrocyte suspension at $37{ }^{\circ} \mathrm{C}$ for $1 \mathrm{~h}$. The samples were centrifuged at $750 \mathrm{~g}$ for $5 \mathrm{~min}$ to remove undamaged erythrocytes. The concentration of released hemoglobin was estimated by reading the absorbance at $545 \mathrm{~nm}$ against the control background lysis solution (erythrocyte suspension with PBS) using the Ultraspec 2100 pro (Amersham Biosciences). The percentage of hemolysis was calculated by normalizing against the absorbance of a mixture of erythrocytes and distilled water at the same ratio, which showed complete hemolysis.

To determine the effect of hemolytic activity on different amounts of red blood cells, rabbit erythrocyte suspensions at different concentrations $(0.2 \%, 0.4 \%, 0.6 \%$, $0.8 \%, 1.0 \%, 1.5 \%, 2.0 \%$ and $2.5 \%$ ) in PBS were used. These diluted cell suspensions were incubated with an equal volume $(1.0 \mu \mathrm{g})$ of $B$. pseudomallei lysate, purified 
BPSL1375 or the mutant protein for $1 \mathrm{~h}$ at $37{ }^{\circ} \mathrm{C}$ and then centrifuged at $750 \mathrm{~g}$ for $5 \mathrm{~min}$ to determine the hemolytic activity. For temperature-dependent studies, equal volumes $(1.0 \mu \mathrm{g})$ of $B$. pseudomallei lysate, purified BPSL1375 and the mutant proteins were incubated with $1 \%$ rabbit erythrocytes at $4{ }^{\circ} \mathrm{C}, 20{ }^{\circ} \mathrm{C}$ and $37{ }^{\circ} \mathrm{C}$ for $1 \mathrm{~h}$ and $60{ }^{\circ} \mathrm{C}$ for $15 \mathrm{~min}$. The mixtures were centrifuged at $750 \mathrm{~g}$ for $5 \mathrm{~min}$ and the residual hemolytic activity of the supernatant was determined.

\section{Scanning electron microscopy}

Sheep erythrocytes (5\%) incubated with BPSL1375 were fixed in $2 \%(\mathrm{v} / \mathrm{v})$ glutaraldehyde, $0.1 \mathrm{M} \mathrm{PBS}(\mathrm{pH} 7.4)$ overnight. Prior to dehydration in ethanol, samples were washed in PBS three times for $10 \mathrm{~min}$. Critical point drying was performed with acetone and sputter-coated with gold-palladium. The samples were observed with a Philips XL30 scanning electron microscope at the Universiti Kebangsaan Malaysia Electron Microscopy Facility.

\section{Additional file}

Additional file 1: Figure A and B. SDS PAGE (A) of soluble fractions for wild type recombinant BPSL1375, R54K mutant, D75E mutant and the corresponding Western blot (B). Lane 1: Broad range marker; Lane 2: E. coli BL21 (DE3) StarTM - IPTG; Lane 3: E. coli BL21 (DE3) StarTM + IPTG; Lane 4: BPSL1375 - IPTG; Lane 5: BPSL1375 + IPTG; Lane 6: R54K - IPTG; Lane 7: R54K + IPTG; Lane 8: D75E - IPTG; Lane 9: D75E + IPTG; Lane 10: purified BPSL1375. Figure C and D. SDS PAGE (C) of soluble fractions for wild type recombinant BPSL1375, D78E mutant, R99K mutant and the corresponding Western blot (D). Lane 1: Broad range marker; Lane 2: E. coli BL21 (DE3) StarTM - IPTG; Lane 3: E. coli BL21 (DE3) StarTM + IPTG; Lane 4: BPSL1375 - IPTG; Lane 5: BPSL1375 + IPTG; Lane 6: D78E - IPTG; Lane 7: D78E + IPTG; Lane 8: R99K - IPTG; Lane 9: R99K + IPTG; Lane 10: purified BPSL1375. Figure E and F. SDS PAGE (E) of soluble fractions for wild type recombinant BPSL1375, E132D mutant, R135K mutant and the corresponding Western blot (F). Lane 1: Broad range marker; Lane 2: E. coli BL21 (DE3) StarTM - IPTG; Lane 3: E. coli BL21 (DE3) StarTM + IPTG; Lane 4: BPSL1375 - IPTG; Lane 5: BPSL1375 + IPTG; Lane 6: E132D - IPTG; Lane 7: E132D + IPTG; Lane 8: R135K - IPTG; Lane 9: R135K + IPTG; Lane 10: purified BPSL1375. (PDF $469 \mathrm{~kb})$

\section{Competing interests}

The authors declare that they have no competing interests.

\section{Authors' contributions}

TLH carried out the PCR, cloning and initial hemolysin assays and SEM sample preparations. LA carried out the mutation, subsequent assays with the mutants, analyzed the data and drafted the manuscript. NAMA drafted the manuscript and carried out the in-vitro assays. RM and SN designed the experiments and drafted the manuscript. MF-R designed the experiments, analyzed the data and drafted the manuscript. All authors read and approved the final manuscript.

\section{Acknowledgements}

The service provided by the Electron Microscopy Facility, Universiti Kebangsaan Malaysia is gratefully acknowledged. The authors thank Prof. David Rice and the late Prof. Peter J. Artymiuk (the University of Sheffield) for useful discussions and comments with regard to this work and an earlier draft manuscript.

\section{Author details}

${ }^{1} S c h o o l$ of Biosciences and Biotechnology, Faculty of Science and Technology, Universiti Kebangsaan Malaysia, 43600 UKM, Bangi, Selangor, Malaysia. ${ }^{2}$ Malaysia Genome Institute, 43000, Kajang, Selangor, Malaysia. ${ }^{3}$ INTI International University, Bandar Baru Nilai, Nilai, Negeri Sembilan 71800, Malaysia. ${ }^{4}$ Institute of Systems Biology, Universiti Kebangsaan Malaysia, 43600 UKM, Bangi, Selangor, Malaysia.

Received: 13 April 2015 Accepted: 13 November 2015

Published online: 23 November 2015

\section{References}

1. Currie BJ, Fisher DA, Howard DM, Burrow JNC, Selvanayagam S, Snelling PL, et al. The epidemiology of melioidosis in Australia and Papua New Guinea. Acta Trop. 2000;74(2-3):121-7.

2. Wiersinga WJ, van der Poll T, White NJ, Day NP, Peacock SJ. Melioidosis: insights into the pathogenicity of Burkholderia pseudomallei. Nat Rev Micro. 2006;4(4):272-82

3. Gauthier YP, Thibault FM, Paucod JC, Vidal DR. Protease production by Burkholderia pseudomallei and virulence in mice. Acta Trop. 2000; 74(2-3):215-20.

4. Korbsrisate S, Tomaras AP, Damnin S, Ckumdee J, Srinon V, Lengwehasatit I, et al. Characterization of two distinct phospholipase $C$ enzymes from Burkholderia pseudomallei. Microbiology. 2007;153(6):1907-15.

5. Burtnick MN, Brett PJ, Harding SV, Ngugi SA, Ribot WJ, Chantratita N, et al. The cluster 1 type $\mathrm{VI}$ secretion system is a major virulence determinant in Burkholderia pseudomallei. Infect Immun. 2011;79(4):1512-25.

6. Scott AE, Burtnick MN, Stokes MG, Whelan AO, Williamson ED, Atkins TP, et al. Burkholderia pseudomallei capsular polysaccharide conjugates provide protection against acute melioidosis. Infect Immun. 2014;82(8):3206-13.

7. Cruz-Migoni A, Hautbergue GM, Artymiuk PJ, Baker PJ, Bokori-Brown M, Chang C-T, et al. A Burkholderia pseudomallei toxin inhibits helicase activity of translation Factor elF4A. Science. 2011:334(6057):821-4.

8. Khoo J-S, Chai S-F, Mohamed R, Nathan S, Firdaus-Raih M. Computational discovery and RT-PCR validation of novel Burkholderia conserved and Burkholderia pseudomallei unique sRNAs. BMC Genomics. 2012;13 Suppl 7:S13.

9. Nandi T, Holden M, Didelot X, Mehershahi K, Boddey JA, Beacham I, et al.: Burkholderia pseudomallei sequencing identifies genomic clades with distinct recombination, accessory and epigenetic profiles. Genome Res. 2014;25:129-41.

10. Rohmer L, Hocquet D, Miller SI. Are pathogenic bacteria just looking for food? Metabolism and microbial pathogenesis. Trends Microbiol. 2011; 19(7):341-8.

11. Pishchany GSE. Taste for Blood: Hemoglobin as a Nutrient Source for Pathogens. PLoS Pathog. 2012;8(3):e1002535

12. Chakraborty T, Kathariou S, Hacker J, Hof H, Huhle B, Wagner W, et al. Molecular analysis of bacterial cytolysins. Rev Infect Dis. 1987;9(Supplement 5):S456-66.

13. Rowe GE, Pellett $\mathrm{S}$, Welch RA. Analysis of toxinogenic functions associated with the RTX repeat region and monoclonal antibody D12 epitope of Escherichia coli hemolysin. Infect Immun. 1994;62(2):579-88.

14. Karch H, Tarr PI, Bielaszewska M. Enterohaemorrhagic Escherichia coli in human medicine. Int J Med Microbiol. 2005;295(6-7):405-18.

15. Oliver JD, Roberts DM, White VK, Dry MA, Simpson LM. Bioluminescence in a strain of the human pathogenic bacterium Vibrio vulnificus. Appl Environ Microbiol. 1986;52(5):1209-11.

16. Kim YR, Kim BU, Kim SY, Kim CM, Na HS, Koh JT, et al. Outer membrane vesicles of Vibrio vulnificus deliver cytolysin-hemolysin VvhA into epithelial cells to induce cytotoxicity. Biochem Biophys Res Commun. 2010;399(4):607-12.

17. Fernández L, Méndez J, Guijarro JA. Molecular virulence mechanisms of the fish pathogen Yersinia ruckeri. Vet Microbiol. 2007;125(1-2):1-10.

18. Radtke ALA, Kelsi L, Davis MJ, DiMagno MJ, Swanson JA, O'Riordan MX. Listeria monocytogenes exploits cystic fibrosis transmembrane conductance regulator (CFTR) to escape the phagosome. Proc Natl Acad Sci. 2011;108(4):1633-8.

19. Hutchison ML, Poxton IR, Govan JR. Burkholderia cepacia produces a hemolysin that is capable of inducing apoptosis and degranulation of mammalian phagocytes. Infect Immun. 1998;66(5):2033-9.

20. Thomson EL, Dennis JJ. A Burkholderia cepacia complex non-ribosomal peptide-synthesized toxin is hemolytic and required for full virulence. Virulence. 2012;3(3):286-98. 
21. Abe M, Nakazawa T. Characterization of hemolytic and antifungal substance, cepalycin, from Pseudomonas cepacia. Microbiol Immunol. 1994;38(1):1-9.

22. Vasil M, Krieg D, Kuhns J, Ogle J, Shortridge V, Ostroff R, et al. Molecular analysis of hemolytic and phospholipase $\mathrm{C}$ activities of Pseudomonas cepacia. Infect Immun. 1990;58(12):4020-9.

23. Ashdown LR, Koehler JM. Production of hemolysin and other extracellular enzymes by clinical isolates of Pseudomonas pseudomallei. J Clin Microbiol. 1990;28(10):2331-4.

24. Häubler S, Nimtz M, Domke T, Wray V, Steinmetz I. Purification and characterization of a cytotoxic exolipid of Burkholderia pseudomallei. Infect Immun. 1998;66(4):1588-93.

25. Haase A, Janzen J, Barret S, Currie B. Toxin production by Burkholderia pseudomallei strains and correlation with severity of melioidosis. J Med Microbiol. 1997;46(7):557-63.

26. Yam H, Abdul Rahim A, Gim Luan O, Samian R, Abdul Manaf U, Mohamad $\mathrm{S}$, et al. Validation of a Burkholderia pseudomallei Hypothetical Protein and Determination of Its Translational Start Codon Using Chromosomal Integration of His-Tag Coding Sequence. Protein J. 2012;31(3):246-9.

27. Holden MTG, Titball RW, Peacock SJ, Cerdeño-Tárraga AM, Atkins T, Crossman LC, et al. Genomic plasticity of the causative agent of melioidosis, Burkholderia pseudomallei. Proc Natl Acad Sci U S A. 2004;101(39):14240-5.

28. Wattam AR, Abraham D, Dalay O, Disz TL, Driscoll T, Gabbard JL, et al.: PATRIC, the bacterial bioinformatics database and analysis resource. Nucleic Acids Res. 2013:42:581-591.

29. Magrane M, UniProt Consortium. UniProt Knowledgebase: a hub of integrated protein data. Database: The Journal of Biological Databases and Curation. 2011; bar009. http://database.oxfordjournals.org/ citmgr?gca $=$ databa\%3B2011\%2F0\%2Fbar009.

30. Berman HM, Westbrook J, Feng Z, Gilliland G, Bhat TN, Weissig H, et al. The Protein Data Bank. Nucleic Acids Res. 2000;28(1):235-42.

31. Gould TA, Schweizer HP, Churchill MEA. Structure of the Pseudomonas aeruginosa acyl-homoserinelactone synthase Lasl. Mol Microbiol. 2004; 53(4):1135-46.

32. Bordo D, Argos P. Suggestions for "safe" residue substitutions in sitedirected mutagenesis. J Mol Biol. 1991;217(4):721-9.

33. Pettersen EF, Goddard TD, Huang CC, Couch GS, Greenblatt DM, Meng EC, et al. UCSF Chimera-A visualization system for exploratory research and analysis. J Comput Chem. 2004;25(13):1605-12.

34. Oberley TD, Duncan JL. Characteristics of streptolysin $\mathrm{O}$ action. Infect Immun. 1971;4(6):683-7.

35. Baida GE, Kuzmin NP. Mechanism of action of hemolysin III from Bacillus cereus. Biochim Biophys Acta Biomembr. 1996;1284(2):122-4.

36. Kretzschmar U, Hammann R, Kutzner H. Purification and characterization of Gardnerella vaginalis hemolysin. Curr Microbiol. 1991:23(1):7-13.

37. Bi S, Wang W, Zhao Y, Ru S, Liu Y. Studies on hemolysis of hemolysin produced by Synechocystis sp. PCC 6803. J Ocean Univ China. 2011;10(4):362-8.

38. Inoue K, Akiyama Y, Kinoshita T, Higashi Y, Amano T. Evidence for a one-hit theory in the immune bactericidal reaction and demonstration of a multihit response for hemolysis by streptolysin $\mathrm{O}$ and Clostridium perfringens theta-toxin. Infect Immun. 1976;13(2):337-44.

39. Winsor GL, Khaira B, Van Rossum T, Lo R, Whiteside MD, Brinkman FSL. The Burkholderia Genome Database: facilitating flexible queries and comparative analyses. Bioinformatics. 2008;24(23):2803-4.

40. Thompson JD, Gibson TJ, Higgins DG. Multiple Sequence Alignment Using ClustalW and ClustalX. Curr Protoc Bioinformatics. 2002: 00: 2.3:2.3.1-2.3.22.

41. Lee S-H, Chong C-E, Lim B-S, Chai S-J, Sam K-K, Mohamed R, et al. Burkholderia pseudomallei animal and human isolates from Malaysia exhibit different phenotypic characteristics. Diagn Microbiol Infect Dis. 2007;58(3):263-70.

42. Richards E, Reichardt M, Rogers S. Preparation of Genomic DNA from Plant Tissue. In: Ausubel FM BR, Kingston RE, Moore DD, Seidman JG, et al. editors. Current Protocols in Molecular Biology. New York: John Wiley \& Sons, Inc; 1994. p. 2.3.1-7.

\section{Submit your next manuscript to BioMed Central and we will help you at every step:}

- We accept pre-submission inquiries

- Our selector tool helps you to find the most relevant journal

- We provide round the clock customer support

- Convenient online submission

- Thorough peer review

- Inclusion in PubMed and all major indexing services

- Maximum visibility for your research

Submit your manuscript at www biomedcentral com/submit 